Si l'on veut savoir comment évoluent les dépenses de santé d'un pays, par comparaison avec d'autres pays, l'utilisation des montants exprimés en monnaies converties, grâce aux taux de change en cours, introduit un paramètre exogène: les taux de change obéissent à des lois du marché financier, qui ne reflètent pas forcément l'économie des pays comparés.

Pour permettre des comparaisons ayant un certain sens, l'OCDE a développé un outil qui permet d'éliminer les variations du marché monétaire, les "parités de pouvoir d'achat " ou PPA.

Les PPA indiquent le montant d'une devise nationale nécessaire pour acquérir une quantité de biens et de services équivalente dans chaque pays à la contrevaleur d'un dollar américain.

Par exemple, le montant du produit intérieur brut (PIB) par tête peut s'exprimer en PPA, ce qui conduit parfois à des classements différents de ceux que permettent les comparaisons par l'intermédiaire des taux de change, mêmes moyens.

PIB par tête en 1988 (exprimé en dollars-P.P.A.)

États-Unis

Canada

19581

Japon

RFA

18289

14268

14243

France

13624

Royaume-Uni $\quad 13521$

Italie

\section{UN NOUVEAU ET BON GĖNE CANDIDAT POUR LE GĖNE DE DÉTERMINATION DU SEXE}

Fin 1987, le groupe de David Page proposait une cartographie précise du locus TDF (testis determining factor) et l'isolement d'un gène candidat (Cell 1987; $51: 1091-104$ ) appelé par la suite ZFY. Récemment, dans un travail conjoint, les groupes de Peter Goodfellow et Marc Fellous concluaient à une localisation plus distale, a priori inconciliable avec celle de Page (Nature 1989 ; 342 : 937-9). Mais dans une lettre à la revue Nature $(1990 ; 346: 279-81)$, ce dernier vient de mettre fin à ce paradoxe en montrant que l'une des anomalies structurales du chromosome $Y$ sur lesquelles reposait sa cartographie est en fait caractérisée par une double délétion : la deuxième délétion nouvellement observée recouvre l'intervalle de localisation proposé par Goodfellow et Fellous. Dans le même numéro de Nature, deux articles consécutifs des équipes de Peter Goodfellow et Robin Lovell-Badge (1990; 346 : 240-4 et 245-50) décrivent l'identification d'un nouveau gène $S R Y$ (sex determining region $Y$, Sry chez la souris). Ce gène répond à toute une série de critères qui en font un candidat TDF des plus crédibles.

- SRY/Sry est conservé sur le chromosome Y de tous les mammifères euthériens analysés.

- SRY/Sry est situé dans l'intervalle de localisation de TDF chez l'homme et de l'équivalent $T d y$ chez la souris. Des souris mutantes, $X Y$ femmes fertiles, présentant une microdélétion du chromosome $Y$, ont notamment perdu Sry alors qu'elles ont conservé $Z f y 1$ et $Z f y 2$, les deux homologues murins de ZFY.

- Un produit de transcription a été observé dans le testicule humain adulte et sur l'embryon de souris, dans les crêtes génitales en voie de différenciation testiculaire (jour 11,5).

- SRY et Sry ont une phase de lecture ouverte dont une partie est fortement conservée. Un motif de 80 acides aminés de la séquence codante de SRY/Sry présente des analogies frappantes avec la protéine du type sexuel Mc de la levure Saccharomyces pombe ainsi qu'avec un domaine de protéines nucléaires non histones du groupe HMG. On pense que ces protéines nucléaires sont susceptibles de se lier à l'ADN par l'intermédiaire de ce domaine appelé boîte HMG. SRY/Sry pourrait donc être une protéine se liant à I'ADN.

II reste maintenant à prouver que ce nouveau gène candidat correspond à $T D F$; par exemple en observant des mutations ponctuelles de $S R Y$ chez des femmes $X Y$ ou des souris transgéniques, portant une copie de Sry, de caryotype XX mais de sexe masculin. 